\title{
Influence of pulse frequency and bias on microstructure and mechanical properties of TiB2 coatings deposited by high power impulse magnetron sputtering
}

Nils Nedfors, Aurelija Mockuté, Justinas Palisaitis, Per O A Persson, Lars-Åke Näslund and Johanna Rosén

Journal Article

Tweet

N.B.: When citing this work, cite the original article.

Original Publication:

Nils Nedfors, Aurelija Mockuté, Justinas Palisaitis, Per O A Persson, Lars-Åke Näslund and Johanna Rosén, Influence of pulse frequency and bias on microstructure and mechanical properties of TiB2 coatings deposited by high power impulse magnetron sputtering, Surface \&amp; Coatings Technology, 2016. 304, pp.203-210.

http://dx.doi.org/10.1016/j.surfcoat.2016.06.086

Copyright: Elsevier

\section{http://www.elsevier.com/}

Postprint available at: Linköping University Electronic Press

http://urn.kb.se/resolve?urn=urn:nbn:se:liu:diva-132332
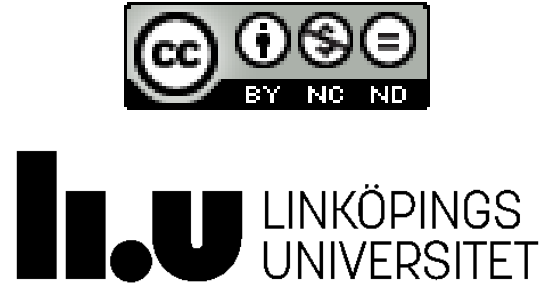


\title{
Influence of pulse frequency and bias on microstructure and mechanical properties of
} $\mathrm{TiB}_{2}$ coatings deposited by high power impulse magnetron sputtering

Nils Nedfors*, Aurelija Mockute, Justinas Palisaitis, Per O. Å. Persson, Lars-Åke Näslund, Johanna Rosen

Thin Film Physics Division, Department of Physics, Chemistry, and Biology, IFM, Linköping University, SE-581 83 Linköping, Sweden

*Corresponding author; phone: +46 13 281232, e-mail: nils.nedfors@liu.se

\begin{abstract}
The high plasma density and large fraction of ionized species created in a high power impulse magnetron sputtering (HiPIMS) discharge add new measures to control the sputtering process. We have studied the sputtering of $\mathrm{TiB}_{2}$ coatings by HiPIMS from a compound target in an industrial system. How the degree of ionized species effects coating microstructure and mechanical properties has been investigated by varying the pulse frequency between $200 \mathrm{~Hz}$ and $1000 \mathrm{~Hz}$ while keeping the average power constant at $2 \mathrm{~kW}$. The coatings have a B/Ti atomic ratio $\geq 2.5$ and a microstructure exhibiting 001 textured nanocolumnar grains with an amorphous B tissue phase in grain boundaries. Lower frequencies provide higher degree of ionization, which does, however, increase the compressive residual stress in the coatings. This results in harder coatings and the highest hardness of $49 \mathrm{GPa}$ is measured for the coating deposited at $200 \mathrm{~Hz}$ (-3.8 GPa residual stress). A change in texture from random orientation to 001 texture is achieved when going from regular dc sputtering to HiPIMS at a floating bias. Superhard ( $\mathrm{H}=43 \mathrm{GPa}) \mathrm{TiB}_{2}$ coatings with a relatively low compressive stress of about -1 GPa can be deposited by HiPIMS at $1000 \mathrm{~Hz}$ using floating bias.
\end{abstract}

Keywords: titanium diboride; hipims; residual stress; texture; mechanical properties;

superhardness 


\section{Introduction}

The high hardness, chemical stability and high melting temperature of titanium diboride $\left(\mathrm{TiB}_{2}\right)$ have attracted a growing interest of this material for protective and wear resistant coatings [1-5]. $\mathrm{TiB}_{2}$ crystallizes in the hexagonal $\mathrm{AlB}_{2}$ structure with close-packed Ti layers alternated with graphite-like B layers. The refractory properties of this material are attributed to strong covalent bonds between the $\mathrm{B}$ atoms within the layer as well as bonds of covalent and ionic nature between the metal and $\mathrm{B}$ layers [6].

There are numerous studies of magnetron sputtered $\mathrm{TiB}_{2}$ coatings in the literature [1-3, 7-12]. Previous depositions are almost exclusively performed using $\mathrm{TiB}_{2}$ compound targets and often results in overstoichiometric coatings, i.e., $\mathrm{B} / \mathrm{Ti}>2[1,3,7,10,11]$. The excess $\mathrm{B}$ segregates to grain boundaries forming an amorphous tissue phase [7, 13]. Coating microstructure and hence properties are heavily influenced by the sputtering parameters and superhardness $\left(\mathrm{H}>40 \mathrm{GPa}\right.$ ) is reported for coatings exhibiting nanocolumnar $\mathrm{TiB}_{2}$ grains with a 001 orientation $[1,2,7]$. The nanocolumnar structure limits the nucleation and slip of dislocations while the tissue phase hinders grain boundary sliding. The high hardness is a result of the combination of these effects [7]. Many studies are therefore devoted to investigate the influence of different sputtering parameters, such as substrate temperature and bias, on the resulting microstructure $[1,2,9,10,12]$.

In high power impulse magnetron sputtering (HiPIMS) short high power pulses are applied to the target. A low duty cycle of the pulses is used to maintain an average power in the same order of magnitude as for conventional dc magnetron sputtering but the peak power is two orders of magnitude higher. This results in a high plasma density and a much larger fraction of ionized species for the HiPIMS process in comparison to dc magnetron sputtering $[14,15]$. The energy available during coating growth can be enhanced by ion bombardment. The flux, nature, and energy of the bombarding ions will influence the microstructure $[16,17]$. Hence, the high fluxes of ionized species available in the HiPIMS discharge add an extra dimension to control the coating microstructure. The only study, to our knowledge, utilizing the HiPIMS technique in the deposition of $\mathrm{TiB}_{2}$ coatings is a recent report by Zhang et al. [3]. One set of HiPIMS discharge parameters was used to investigate the substrate bias and temperature effect on the coating microstructure and mechanical properties. They see a strong dependency on texture with applied bias in accordance with previous studies on the influence of bias for magnetrons sputtered $\mathrm{TiB}_{2}$ coatings $[2,10]$. However, the influence of different HiPIMS sputtering parameters, such as pulse frequency, is not investigated.

A higher peak current results in a higher fraction of ionized sputtered species $[17,18]$. The peak current may be increased by keeping the average power and pulse length constant while reducing the pulse frequency. It is thereby possible to control the flux of ionized species reaching the substrate by varying the frequency. The effect of HiPIMS pulse frequency on coating properties for the sputtering of $\mathrm{ZrB}_{2}$ coatings has been reported by Samuelsson et al. [19]. The authors observed a change in residual stress from tensile to compressive and reduction in size of 001 textured $\mathrm{ZrB}_{2}$ grains when the frequency is decreased from $900 \mathrm{~Hz}$ to $300 \mathrm{~Hz}$ (i.e., ion density flux towards the substrate is increased). Furthermore, a denser morphology is observed for coatings deposited by HiPIMS, especially at lower frequencies, in comparison to a $\mathrm{ZrB}_{2}$ coating deposited by dc magnetron sputtering.

The aim with this study is to investigate the ion-flux effect on the resulting microstructure and mechanical properties of $\mathrm{TiB}_{2}$ coatings deposited by HiPIMS. A variation in the degree of ionized species is achieved by changing the pulse frequency. The HiPIMS sputtered $\mathrm{TiB}_{2}$ coatings will be compared to coatings deposited using conventional dc magnetron sputtering. 
In addition, to study the effect of ion energy, two different biasing modes are used in the coating depositions.

\section{Experimental}

The $\mathrm{TiB}_{2}$ coatings were synthesized in a high vacuum CC800/9 CemeCon AG deposition system by sputtering from an $8.8 \times 50 \mathrm{~cm}^{2}$ compound $\mathrm{TiB}_{2}$ target supplied by CemeCon AG. Prior to deposition the chamber was degassed by applying a power of $8.8 \mathrm{~kW}$ to each of two resistive heaters for $30 \mathrm{~min}$. Thereafter the power was reduced to $8.0 \mathrm{~kW}$ for $60 \mathrm{~min}$ resulting in a substrate temperature of approximately $500^{\circ} \mathrm{C}$ and a base pressure of $\leq 1.0 \mathrm{mPa}$. The heating power was kept at $8.0 \mathrm{~kW}$ throughout the deposition. $10 \times 10 \mathrm{~mm}^{2} \mathrm{Al}_{2} \mathrm{O}_{3}(001)$ and 10 $\mathrm{x} 10 \mathrm{~mm}^{2} \mathrm{Si}(001)$ substrates were mounted by clips along a line in parallel with the long side of the target and facing the target surface at a target-to-substrate distance of $60 \mathrm{~mm}$. The $\mathrm{Al}_{2} \mathrm{O}_{3}$ substrates were primarily chosen for nanoindentation measurements since their high hardness and elastic modulus matches the mechanical properties of $\mathrm{TiB}_{2}$ while Si substrates were chosen for electron microscopy studies. An Ar process pressure of $0.4 \mathrm{~Pa}$ was used for all depositions. The $\mathrm{TiB}_{2}$ cathode was operated in HiPIMS mode applying an average power of $2 \mathrm{~kW}$ and a pulse length of $200 \mu \mathrm{s}$. Two series of coatings were deposited using four different frequencies: $200 \mathrm{~Hz}, 300 \mathrm{~Hz}, 500 \mathrm{~Hz}$, and $1000 \mathrm{~Hz}$. Two different modes of bias were used; floating or pulsed bias. In the pulsed mode $-60 \mathrm{~V}$ was applied synchronous with the $200 \mu$ s HiPIMS pulse and with floating potential between the pulses. For comparison, $\mathrm{TiB}_{2}$ coatings were also deposited using dc magnetron sputtering at a power of $2 \mathrm{~kW}, 0.4 \mathrm{~Pa}$ Ar process pressure, the same substrate temperature, and with both $-60 \mathrm{~V}$ and floating bias.

X-ray diffraction (XRD) $\theta$-2 $\theta$ measurements were performed using a PANalytical Empyrean diffractometer with $\mathrm{Cu} \mathrm{K \alpha}$ radiation in parallel beam geometry. The coating densities were obtained from simulated X-ray reflectometry (XRR) data fitted to measured XRR data using X'Pert Reflectivity v. 1.3 software from PANalytical. A Philips X'pert diffractometer with Cu $\mathrm{K} \alpha$ radiation in parallel beam geometry was used for the XRR measurements.

Elemental composition and chemical bonding state of the coatings were acquired using a Kratos Analytical instrument AXIS Ultra DLD X-ray photoelectron spectroscopy (XPS) system with monochromatic $\mathrm{Al} \mathrm{K} \alpha$ radiation. Prior to analysis surface contamination was removed through $120 \mathrm{~s}$ sputter-etch using $500 \mathrm{eV} \mathrm{Ar}{ }^{+}$ions over an area of $3 \times 3 \mathrm{~mm}^{2}$. The XPS binding energy scale was calibrated against the Fermi-edge $\left(E_{f}\right)$ of each sample, which was set to a binding energy of $0.0 \mathrm{eV}$. The pure B reference sample (99.6\%) was provided by Goodfellow Cambridge Ltd. The XRD, XRR, and XPS analysis were carried out on coatings deposited on $\mathrm{Al}_{2} \mathrm{O}_{3}(001)$ substrates.

A Zeiss LEO 1550 scanning electron microscopy (SEM) was used to determine coating thicknesses from cross-sectional samples of coatings deposited on the Si substrates. Crosssection samples for transmission electron microscopy (TEM) investigations were prepared by traditional methods including cutting, gluing and polishing followed by $\mathrm{Ar}^{+}$ion milling at 5 $\mathrm{keV}$ and $5^{\circ}$ angle from both sides in a Gatan precision ion polishing system (PIPS). A final low energy milling step was applied at $2 \mathrm{keV}$ in order to minimize the surface damage. The plan-view TEM samples were prepared by cutting $3 \mathrm{~mm}$ discs with an ultrasonic disc-cutter, followed by mechanical polishing and ion milling from the substrate side in line with the cross-sectional samples. TEM and scanning TEM high angle annular dark field (STEMHAADF) imaging was performed in the double-corrected Linköping FEI Titan ${ }^{3} 60-300$, operated at $300 \mathrm{kV}$. Electron diffraction was recorded using a FEI Tecnai G2 TF 20 UT, operated at $200 \mathrm{kV}$. 
Residual stress in the coatings was determined from the curvature of the $\mathrm{Al}_{2} \mathrm{O}_{3}$ substrates. The curvatures were obtained from XRD rocking curve measurements of the substrate $\mathrm{Al}_{2} \mathrm{O}_{3}$ (001) peak at five different spots over the substrate using a PANalytical Empyrean instrument with $\mathrm{Cu} \mathrm{K} \alpha$ radiation and high-resolution optics. The Stoney equation was used to calculate the in-plane biaxial stress in the coating, $\sigma_{c}$, from the substrate curvature $R$ [20]:

$\sigma_{c}=M_{s} \frac{h_{s}^{2}}{6 h_{c} R}$

Where $h_{s}$ and $h_{c}$ are the thicknesses of the substrate and the coating, respectively. For an elastic isotropic substrate the biaxial elastic modulus $M_{s}=E /(1-v)$ where $E$ is the elastic modulus and $v$ is the Poisson's ratio of the substrate. The $\mathrm{Al}_{2} \mathrm{O}_{3}$ substrate is elastically anisotropic and the biaxial modulus, $M^{\mathrm{Al} 2 \mathrm{O} 3}$, is calculated using the stiffness matrix for $\mathrm{Al}_{2} \mathrm{O}_{3}$ and following the method employed by Janssen et al. for $\mathrm{Si}(001)$ substrates [20, 21]:

$M_{(001)}^{A l_{2} O_{3}}=C_{11}+C_{12}-2 \frac{C_{13}^{2}}{C_{33}}$

To consider the $\mathrm{Al}_{2} \mathrm{O}_{3}$ substrate to be elastically isotropic in the plane parallel to the film (i.e., the 001-plane), the small $C_{14}$ term in the stiffness matrix had to be neglected ( $<5 \%$ of the largest term, c.f. $C_{14}=22.9 \mathrm{GPa}$ and $C_{33}=501.8 \mathrm{GPa}$ [22]). A biaxial modulus of $M_{s}=605.5$ $\mathrm{GPa}$, obtained from equation (2) with values for the elastic constants $C_{\mathrm{ij}}$ from ref. [22], is used in the calculations of residual stress.

Mechanical properties of the coatings deposited on the $\mathrm{Al}_{2} \mathrm{O}_{3}$ substrates were obtained using a CSIRO UMIS nanoindenter with a diamond Berkovich tip calibrated against a fused silica reference sample. Hardness and reduced elastic modulus were calculated from the loaddisplacement curves according to the Oliver and Pharr method [23]. About 50 indents were recorded for each sample at an increasing load from 3 to $27 \mathrm{mN}$, the highest load results in a penetration depth of about $200 \mathrm{~nm}$. The elastic modulus of the coating was calculated from the reduced elastic modulus using an elastic modulus of $1141 \mathrm{GPa}$ for the diamond indenter and Poisson's ratios of 0.07 and 0.12 for the indenter and the $\mathrm{TiB}_{2}$ coatings, respectively [23, 25].

\section{Results}

The target peak current at different pulse frequencies and biasing modes is summarized in Table I. Since the average power is kept at $2 \mathrm{~kW}$, the peak current (along with the pulse energy) is reduced from $520 \mathrm{~A}$ to $40 \mathrm{~A}$ as pulse frequency is increased from $200 \mathrm{~Hz}$ to 1000 $\mathrm{Hz}$. The peak current to the substrate table is an indirect measure of the ion-flux to the substrate. A decrease from $17 \mathrm{~A}$ to $12 \mathrm{~A}$ was measured when the frequency was increased from $200 \mathrm{~Hz}$ to $1000 \mathrm{~Hz}$ in $-60 \mathrm{~V}$ bias mode. It should be noted that the substrate table is much larger in size compared to the substrates. Therefore, the absolute values of the peak current to the substrate table are of no interest. Nevertheless, the trend shows that the ion-flux to the substrates follows the target peak current with a maximum in target peak current and substrate peak current at the lowest frequency of $200 \mathrm{~Hz}$. The same trend has previously been reported for HiPIMS sputtering of $\mathrm{Ti}$ and $\mathrm{ZrB}_{2}$ targets [17-19]. No significant difference is observed for the variation in peak current to the substrate table with pulse frequency between the different bias modes. It should be noted that the deposition rate, a measure of the total flux of sputtered species to the substrate, decreases with the reduction in pulse frequency (see Table I). It can thus be concluded that the ion-to-neutral ratio increases with the decrease in pulse frequency considering the simultaneous increase in ion-flux and decrease in total flux of species to the substrate with the frequency reduction. 
Table I. Sputtering parameters, thickness, and density of the studied coatings. The deposition rates marked with an asterix $(*)$ is calculated from coatings deposited using the exact same sputtering process except the deposition time, which were shortened to one fifth.

\begin{tabular}{|l|l|l|l|l|l|l|}
\hline Sample & $\begin{array}{l}\text { Pulse } \\
\text { frequency } \\
{[\mathrm{Hz}]}\end{array}$ & Bias mode & $\begin{array}{l}\text { Cathode } \\
\text { peak current } \\
{[\mathrm{A}]}\end{array}$ & $\begin{array}{l}\text { Deposition } \\
\text { rate } \\
{[\mathrm{nm} / \mathrm{min}]}\end{array}$ & $\begin{array}{l}\text { Coating } \\
\text { thickness } \\
{[\mu \mathrm{m}]}\end{array}$ & $\begin{array}{l}\text { Density } \\
{\left[\mathrm{g} / \mathrm{cm}^{3}\right]}\end{array}$ \\
\hline 1 & 200 & $-60 \mathrm{~V}$ & 520 & $22^{*}$ & 1.76 & 4.40 \\
\hline 2 & 300 & $-60 \mathrm{~V}$ & 292 & $22^{*}$ & 1.10 & 4.31 \\
\hline 3 & 500 & $-60 \mathrm{~V}$ & 132 & $34^{*}$ & 1.02 & 4.19 \\
\hline 4 & 1000 & $-60 \mathrm{~V}$ & 49 & 45 & 0.99 & 4.16 \\
\hline 5 & DC & $-60 \mathrm{~V}$ & - & 53 & 0.80 & 4.18 \\
\hline 6 & 200 & Float & 520 & $24^{*}$ & 1.92 & 4.36 \\
\hline 7 & 300 & Float & 280 & $29 *$ & 1.45 & 4.33 \\
\hline 8 & 500 & Float & 140 & 38 & 1.14 & 4.25 \\
\hline 9 & 1000 & Float & 48 & 49 & 1.08 & 4.18 \\
\hline 10 & DC & Float & - & 60 & 0.90 & 4.12 \\
\hline
\end{tabular}

The chemical composition of the different coatings measured by XPS is plotted in Fig. 1. Only minor variations are seen in the $\mathrm{B}$ and $\mathrm{Ti}$ content with the variation in pulse frequency. The $\mathrm{B} / \mathrm{Ti}$ atomic ratios are in the range 2.4 to 2.8, i.e., the coatings are overstoichiometric, which is typical for coatings deposited by magnetron sputtering from stoichiometric $\mathrm{TiB}_{2}$ targets $[1,3,7,11]$. Neidhardt et al. have performed a comprehensive study of the various mechanisms influencing the composition of coatings deposited from a $\mathrm{TiB}_{2}$ target [11]. The different mass and scattering cross-section of $\mathrm{B}$ and Ti results in different angular and energetic distributions as well as gas phase scattering properties. As a consequence, the B/Ti ratio mainly depends on process pressure as well as target-to-substrate distance and angle [11]. Contaminations of oxygen, carbon, and nitrogen were detected in all coatings. Nitrogen is most probably present as a residual gas in the deposition chamber. The $\mathrm{O}$ and $\mathrm{C}$ contamination occurs most probably after air exposure considering the variation in level of contamination among the coatings with the highest $\mathrm{O}$ contents for the coatings of lowest density (see Table I).

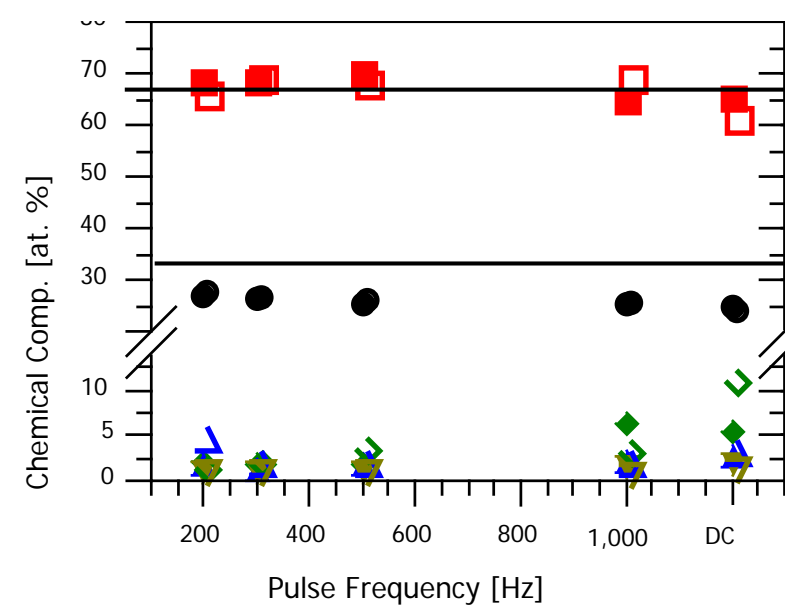

Figure 1. Chemical composition acquired using XPS. Filled and unfilled symbols represent coatings deposited using $-60 \mathrm{~V}$ and floating bias, respectively. The dotted lines mark the B and Ti composition for stoichiometric $\mathrm{TiB}_{2}$. Note the break in the chemical composition axis. 

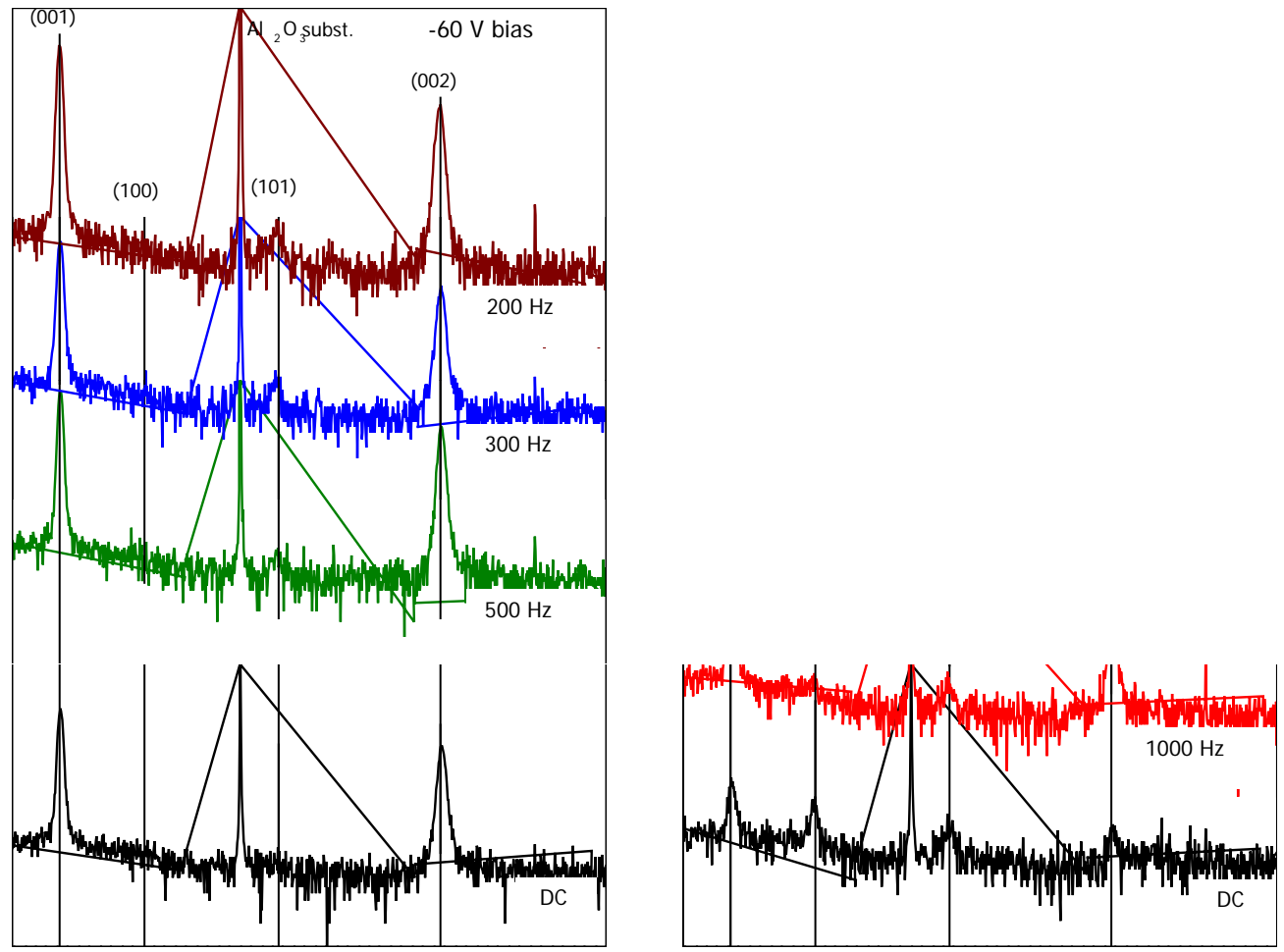

Figure 2. X-ray diffraction $\theta-2 \theta$ scans of the two series of coatings sputtered using different bias modes: $-60 \mathrm{~V}$ bias applied in synchronous with the HiPIMS pulse (left) and floating bias (right). Vertical lines mark reference positions of diffraction peaks from ref. [26].

Coating thicknesses determined from SEM cross-sections, calculated deposition rates and densities determined from XRR measurements are presented in Table I. The coatings deposited on Si by HiPIMS at the lower frequencies have delaminated from the substrates. These depositions were repeated using the exact same process parameters except for the deposition time, which was shortened to one fifth in order to reduce the stress accumulated in the coatings and thus improve adhesion on Si. Deposition rates calculated from these coatings are marked with an asterix in Table I. The deposition rates are $15 \%$ (-60 V bias) and $18 \%$ (floating bias) lower for the HiPIMS depositions at $1000 \mathrm{~Hz}$ compared to the dc depositions. The rates are further reduced by a factor of two with the decrease in pulse frequency from $1000 \mathrm{~Hz}$ to $200 \mathrm{~Hz}$. The coating density increases with the decrease in pulse frequency and the highest density of $4.4 \mathrm{~g} / \mathrm{cm}^{3}$ is measured for the coating deposited at $200 \mathrm{~Hz}$ with a $-60 \mathrm{~V}$ bias. The highest density is $97 \%$ of the theoretical density for stoichiometric $\mathrm{TiB}_{2}$, which is $4.53 \mathrm{~g} / \mathrm{cm}^{3}$. No significant difference is observed between the coatings sputtered using HiPIMS with different bias modes. The dc sputtered coating with a $-60 \mathrm{~V}$ bias has a density in the same range as the coatings deposited using HiPIMS with the higher frequencies and the same bias mode. For the floating bias, a slightly lower density is measured for the dc sputtered coating compared to the coating deposited using HiPIMS at $1000 \mathrm{~Hz}$ (c.f. 4.18 $\mathrm{g} / \mathrm{cm}^{3}$ for $1000 \mathrm{~Hz}$ and $4.12 \mathrm{~g} / \mathrm{cm}^{3}$ for $\mathrm{dc}$ ).

Figure 2 shows XRD $\theta-2 \theta$ diffractograms of the coatings deposited using different pulse frequencies and bias mode. All peaks, except the substrate $\mathrm{Al}_{2} \mathrm{O}_{3}$ peak at $41.7^{\circ}$, can be assigned to the $\mathrm{AlB}_{2}$ structured $\mathrm{TiB}_{2}$ phase [26]. A strong 001 texture is evident for all samples except for the coating deposited using dc sputtering with a floating bias, where 
significant contributions from (100) and (101) planes are apparent. The 001-peak shifts slightly to lower $2 \theta$ values with the decrease in pulse frequency for both series of coatings. This shift corresponds to an expansion of the c-lattice parameter from c $=3.212 \AA$ at $1000 \mathrm{~Hz}$ to $\mathrm{c}=3.221 \AA$ at $200 \mathrm{~Hz}$ for the $-60 \mathrm{~V}$ bias series and from $\mathrm{c}=3.214 \AA$ to $\mathrm{c}=3.222 \AA$ for the floating bias series at the same frequencies, see Fig. 3 . The width of the diffraction peaks is a measure of the $\mathrm{TiB}_{2}$ grain size in the growth direction and level of micro strain in the grains. No frequency dependence is seen in the variation of 001-peak full width at half maximum (FWHM), which is between $0.39^{\circ}-0.44^{\circ}$. The dc sputtered coating using floating bias has a broader 001-peak with a FWHM of $0.59^{\circ}$.

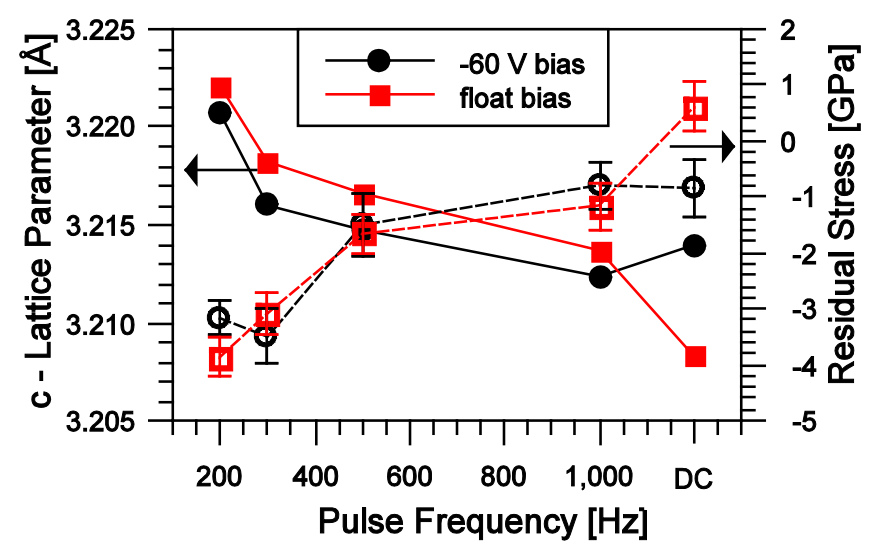

Figure 3 .Left y-axis and filled symbols: variation in lattice parameter c with the HiPIMS pulse frequency for the two series of coatings. The lattice parameter is calculated from the 001 diffraction peak (see Fig. 2). Right y-axis and unfilled symbols: variation in biaxial in-plane residual stress, determined from the curvature of the $\mathrm{Al}_{2} \mathrm{O}_{3}$ substrate, with pulse frequency. The DC label on the pulse frequency axis denotes the samples deposited using regular dc magnetron sputtering.

TEM studies were carried out on two coatings, deposited using dc magnetron sputtering and HiPIMS at a frequency of $200 \mathrm{~Hz}$, both at a $-60 \mathrm{~V}$ bias. Cross-sectional TEM images of the former sample reveal a dense columnar structure, as shown in Fig. 4a and b. The $\mathrm{TiB}_{2}$ columnar grains are $5-10 \mathrm{~nm}$ wide and extend throughout the coating thickness. A 001texture, consistent with the $\theta-2 \theta$ XRD measurement in Fig. 2, is seen in the electron diffraction (ED) pattern shown as an inset in Fig. 4a. A fine grained microstructure, polycrystalline according to ED measurements, is seen closest to the substrate. This structure develop in to the 001 textured columnar structure after about $200 \mathrm{~nm}$. The plan-view projection in Fig. 4c corroborates a microstructure of narrow columnar grains. The lines crossing the grains indicate the presence of stacking faults (marked with arrows in Fig. 4c). Furthermore, it seems that the grains are encapsulated by a thin $(1-2 \mathrm{~nm})$ amorphous tissue phase, which is especially evident in the STEM image (Fig. 4d) where regions containing light elements appear darker. These dark regions can also be interpreted as an under-dense structure, however, according to previous studies of $\mathrm{TiB}_{2}$ coatings, a B-rich tissue phase is expected for overstoichiometric coatings $[7,13]$. Consequently, the dark regions around the bright $\mathrm{TiB}_{2}$ grains are interpreted as a $\mathrm{B}$-rich tissue phase. It is thus confirmed that the coatings exhibit a comparable microstructure to previously reported dc sputtered 001 textured $\mathrm{TiB}_{2}$ coatings [7]. Figure 5a and b show cross-sectional TEM images of the coating deposited using HiPIMS and a bias of $-60 \mathrm{~V}$. This sample exhibits a dense columnar structure as well, though, a sharp line can be seen across the sample at about $2 / 3$ of the total thickness (highlighted with an arrow in Fig. 5a). From high magnification images (not shown) it is determined that this line is an amorphous interlayer and only a few nanometers thick. In addition, EDX analysis shows that the interlayer contains $\mathrm{Si}, \mathrm{Cr}, \mathrm{Fe}, \mathrm{Al}$, and $\mathrm{O}$ and is deficient 
in $\mathrm{Ti}$ and $\mathrm{B}$. The interlayer is most probably caused by arcing during the HiPIMS process resulting in the sputtering of contaminating species from the target vicinity. Similar interlayers, mainly containing $\mathrm{Si}$ and $\mathrm{O}$ are also seen close to the substrate. A polycrystalline fine grained structure is also seen for this coating, near the substrate as well as after the amorphous interlayer at about $2 / 3$ of the total thickness. It extends in the growth direction to a thickness of $300-400 \mathrm{~nm}$ from the substrate and to about $150 \mathrm{~nm}$ from the interlayer. The plan-view image in Fig. 5 c resembles the structure of the dc sputtered coating with grains 5 $10 \mathrm{~nm}$ in diameter and with stacking faults. More notably, the amorphous tissue phase is significantly reduced in Fig. 5c such that only vague dark regions are seen between the grains in the STEM image (see Fig. 5d), which may originate from interface strain at the grain boundaries. Nevertheless, the XPS analysis presented below indicate the existence of a B-rich tissue phase also for this coating.
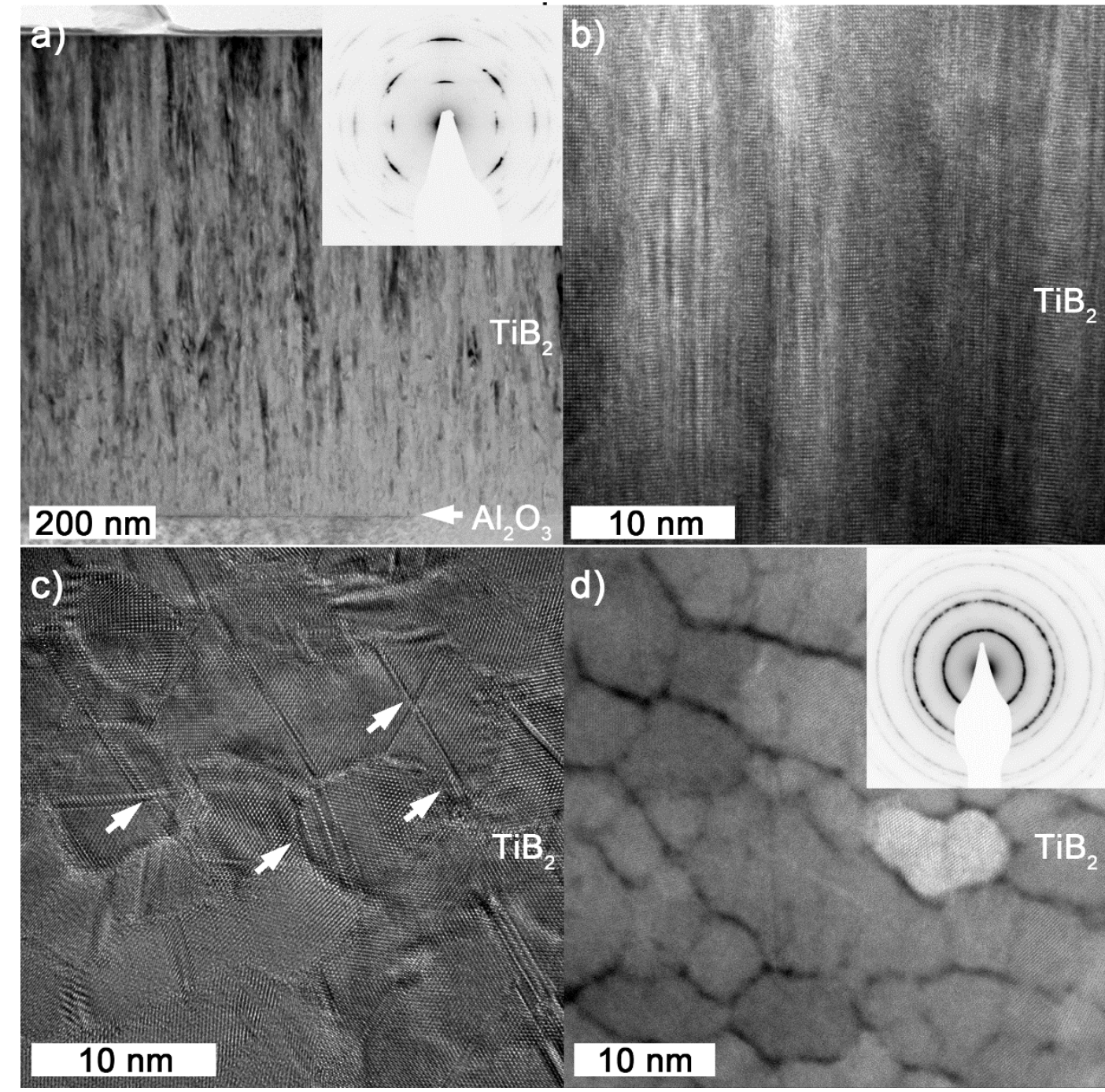

Figure 4. TEM images of the coating deposited using dc magnetron sputtering and -60 V bias: crosssectional bright field TEM, (a) overview and (b) region close to film/substrate interface at higher magnification, as well as plan-view (c) high-resolution TEM and (d) STEM. The insets in (a) and (d) show corresponding electron diffraction patterns. The arrows in (c) indicate stacking faults. 

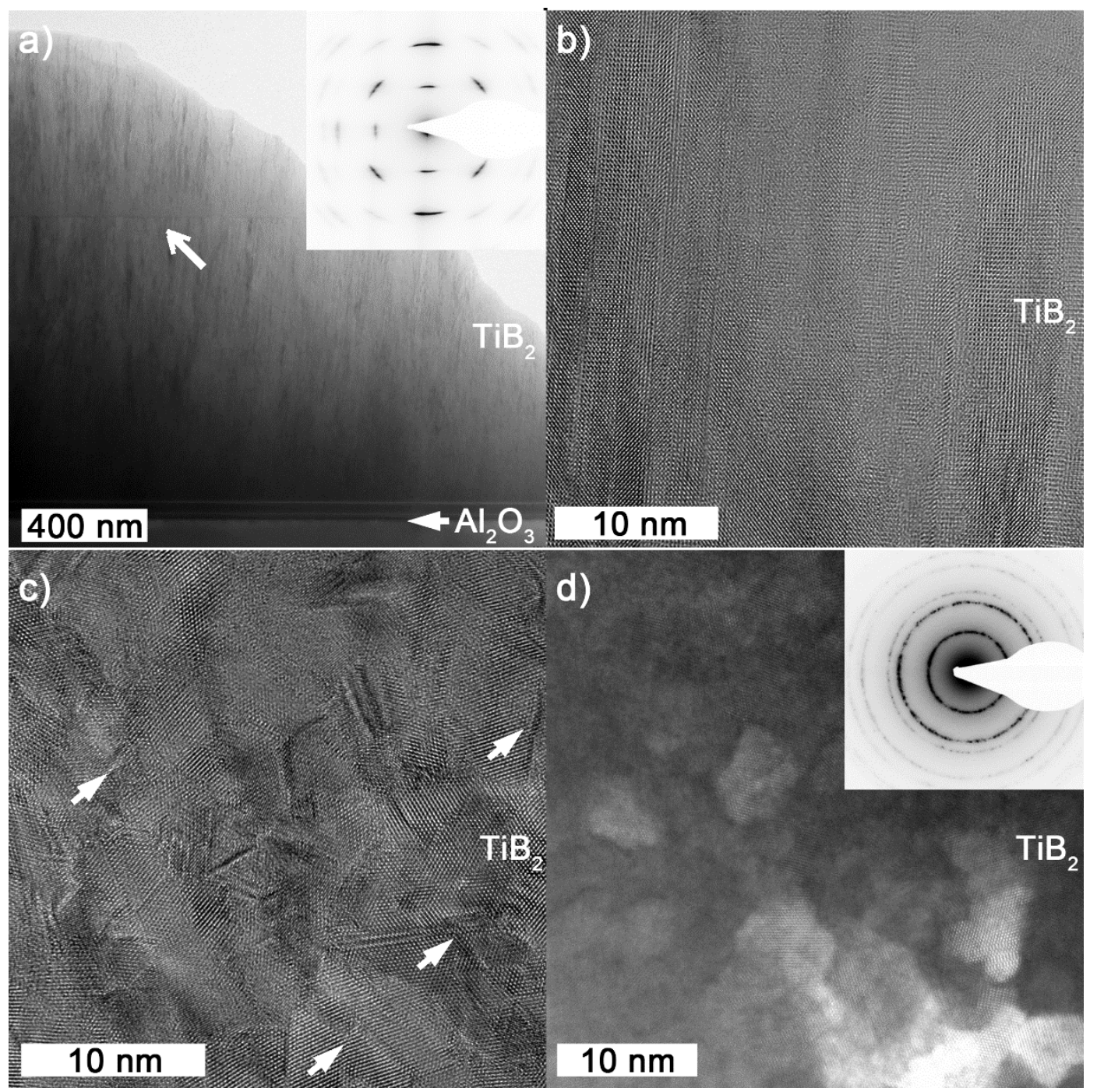

Figure 5. TEM images of the coating deposited using HiPIMS at a frequency of $200 \mathrm{~Hz}$ and $-60 \mathrm{~V}$ bias: cross-sectional bright field TEM (a) overview and (b) region close to film/substrate interface at higher magnification as well as (c) plan-view high-resolution TEM and (d) STEM. The inset in (a) and (c) shows a corresponding electron diffraction pattern. The arrow in (a) points to an interface seen across the coating and the arrows in (c) indicate stacking faults.

The XPS B1s spectra of the coatings deposited using dc sputtering and HiPIMS at a frequency of $200 \mathrm{~Hz}$, both with a $-60 \mathrm{~V}$ bias, are shown in Fig. 6. The B1s spectra from all other samples are similar (not shown). The spectra in Fig. 6 are therefore considered representative for all coatings. The main peak at $187.9 \mathrm{eV}$ is attributed to $\mathrm{B}$ in the $\mathrm{TiB}_{2}$ phase $[27,28]$. The TEM analysis implied the existence of a $\mathrm{B}$-rich tissue phase in the $\mathrm{TiB}_{2}$ grain boundaries and a contribution from $\mathrm{B}-\mathrm{B}$ bonds is therefore expected in the B1s spectra. A reference spectrum for B-B bonds was obtained by measuring a pure bulk B sample. Parameters from this reference spectrum were added as a B-B contribution in the B1s spectra in Fig. 6. The contribution from this peak, i.e., the amount of $\mathrm{B}$ tissue phase in the coatings, decreases slightly with the decrease in $\mathrm{B} / \mathrm{Ti}$ ratio. This is obvious in Fig. 6 where the smaller contribution from B-B bonds for the coating deposited by HiPIMS at $200 \mathrm{~Hz}$ is explained by a lower $\mathrm{B} / \mathrm{Ti}$ ratio of 2.5 for this coating compared to 2.6 for the dc sputtered coating. The smaller contribution from B-B bonds for the coating deposited by HiPIMS agrees with the thinner B-tissue phase barely seen by TEM for this coating. In addition, a third peak had to be included at $187.4 \mathrm{eV}$ in order to achieve a good fit. This peak is attributed to B bonded in the 
$\mathrm{TiB}_{2}$ phase but situated at the interface between the grains and the $\mathrm{B}$ tissue phase. The

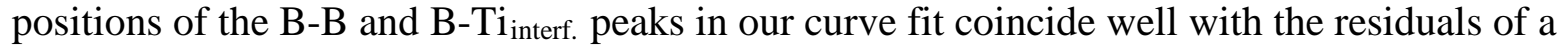
B1s single peak curve fit for an overstoichiometric $\mathrm{TiB}_{2}$ coating by Pfohl et al. [5]. The amount of $\mathrm{B}$ atoms bonded in the $\mathrm{TiB}_{2}$ phase is determined by comparing the areas under the peaks in Fig. 6. By relating this number to the chemical composition of the coating, the stoichiometry of the $\mathrm{TiB}_{2}$ grains, i.e., $\mathrm{x}$ in $\mathrm{TiB}_{\mathrm{x}}$, is calculated. The stoichiometry varies between 2.0 and 2.1 for all coatings, which is consistent with the Ti-B phase diagram and the narrow single-phase composition range of $\mathrm{TiB}_{2}$ [29]. In addition to the three main peaks around $188 \mathrm{eV}$ there is a small peak at $192.8 \mathrm{eV}$ assigned to $\mathrm{B}-\mathrm{O}$ bonds in the spectrum from the coating deposited using dc magnetron sputtering and floating bias.

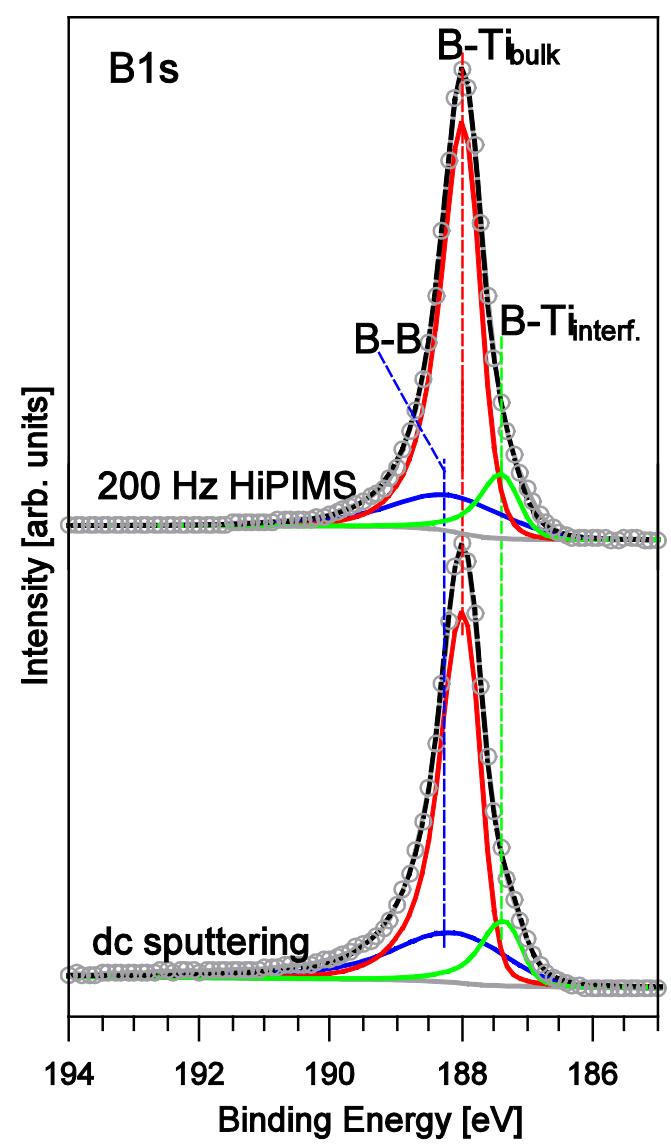

Figure 6. High-resolution XPS B1s spectra of the coatings deposited using dc sputtering and HiPIMS at a frequency of $200 \mathrm{~Hz}$, both with a $-60 \mathrm{~V}$ bias. The spectra have been acquired after 2 min of 500 $\mathrm{eV} \mathrm{Ar}^{+}$ion etching. The grey circles mark the measured raw intensity, while the solid lines represent $\mathrm{B}$ in $\mathrm{TiB}_{2}$ (red), B-B bonds (blue), interface $\mathrm{TiB}_{2}$ (green), the Shirley background (grey), and the sum of all fitted components (black). In the curve fitting of the spectrum the bulk $\mathrm{TiB}_{2}$ and interface $\mathrm{TiB}_{2}$ peaks were confined to have the same shape and FWHM while the curve of the B-B bonds was confined to fit a pure B reference sample. 
Residual stress in the coatings was calculated from the curvature of the $\mathrm{Al}_{2} \mathrm{O}_{3}(001)$ substrates, see Fig. 3. All coatings deposited by HiPIMS are in a compressed stress state. The magnitude of the stress increases with the decrease in pulse frequency and the highest values of -3.2 GPa (-60 V bias) and -3.8 GPa (floating bias) are measured for the coatings deposited using $300 \mathrm{~Hz}$ and $200 \mathrm{~Hz}$, respectively. For the $-60 \mathrm{~V}$ bias series both the dc sputtered coating and the HiPIMS sputtered coating at $1000 \mathrm{~Hz}$ have a stress of $-0.7 \mathrm{GPa}$. A larger difference is detected in the floating bias series where the dc sputtered coating has a tensile stress of 0.6 GPa while the coating deposited using HiPIMS at $1000 \mathrm{~Hz}$ has a compressive stress of -1.0 GPa.

Hardness and elastic modulus, determined from nanoindentation data, are displayed versus the pulse frequency in Fig. 7 and Fig. 8, respectively. Corresponding values for the coatings deposited using dc magnetron sputtering are also included in the figures. The highest hardness for both biasing modes is achieved at the lowest frequency of $200 \mathrm{~Hz}$. For the series of coatings deposited using floating bias the hardness is reduced from $49 \mathrm{GPa}$ to $43 \mathrm{GPa}$ with the increase in pulse frequency from $200 \mathrm{~Hz}$ to $1000 \mathrm{~Hz}$. The same trend is observed for the series with a $-60 \mathrm{~V}$ bias, although the hardness is slightly lower for all samples compared to the floating bias series (500 Hz is an exception but within the error limit). These values are within the typical range for nanocolumnar 001 textured $\mathrm{TiB}_{2}$ coatings, although hardness of about $60 \mathrm{GPa}$ has been reported [1-3, 7]. For the elastic modulus only minor variations with the frequency are seen, considering the magnitude of the standard deviation. The elastic modulus of the coatings deposited with a $-60 \mathrm{~V}$ bias is about $480 \mathrm{GPa}$ while the series with floating bias has a slightly lower modulus of about $460 \mathrm{GPa}$. These values are consistent with previous studies of dc sputtered $\mathrm{TiB}_{2}$ coatings [1, 2]. However, our elastic modulus values are considerably higher compared to the values reported by Zhang et al. for $\mathrm{TiB}_{2}$ coatings deposited by HiPIMS [3]. It should be noted that accurate elastic modulus measurements for coatings of this thickness $(1-2 \mu \mathrm{m})$ require sharp indenters and this is reflected in the large error bars in Fig. 8 [24]. The dc sputtered sample with a $-60 \mathrm{~V}$ bias has the same hardness of $42 \mathrm{GPa}$ as the coating sputtered using HiPIMS with a frequency of $1000 \mathrm{~Hz}$. However, the elastic modulus is slightly higher for the dc sputtered sample. The other coating deposited using dc sputtering but with a floating bias stands out from the rest of the samples with a hardness of only $36 \mathrm{GPa}$. On the other hand, the elastic modulus of $452 \mathrm{GPa}$ follows the trend seen for the other coatings deposited using floating bias.

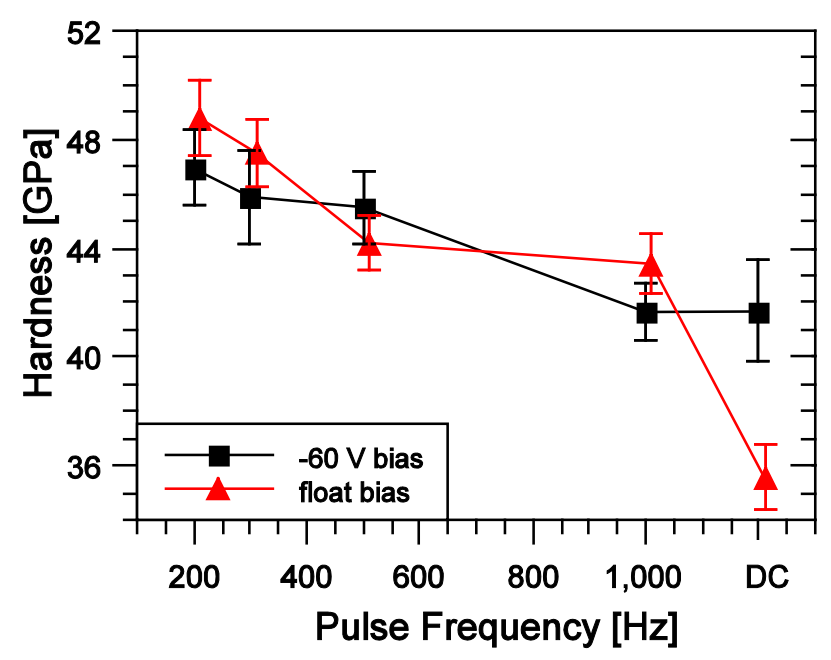

Figure 7. Variation in hardness with pulse frequency for the two series of coatings deposited using different biasing modes. The DC label on the pulse frequency axis denotes the samples deposited using regular dc magnetron sputtering. 


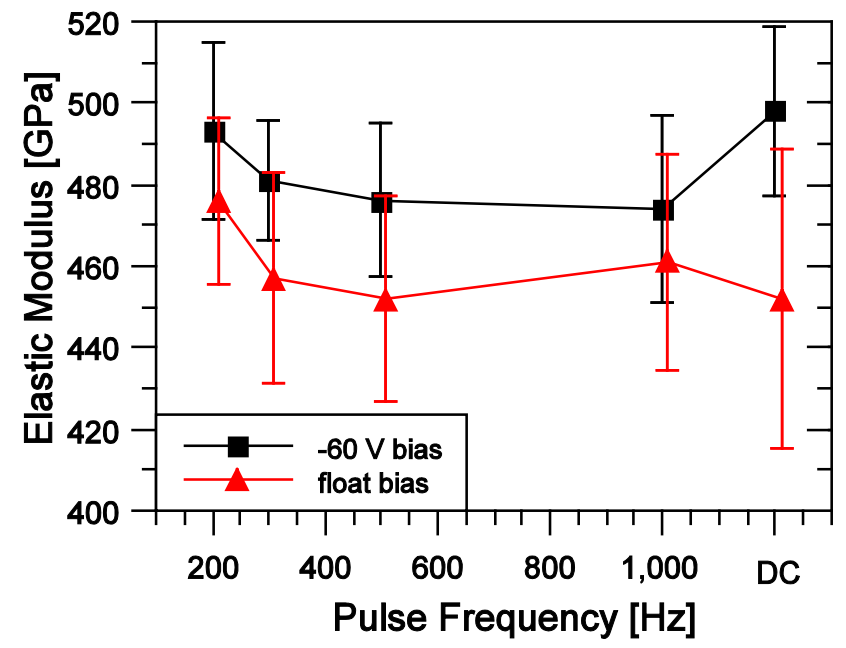

Figure 8. Variation in elastic modulus with pulse frequency for the two series of coatings deposited using different biasing modes. The DC label on the pulse frequency axis denotes the samples deposited using regular dc magnetron sputtering.

\section{Discussion}

The total ion-flux to the substrate and hence the energy available at the coating surface for film growth varies with the pulse frequency. Nevertheless, all HiPIMS coatings, independent of frequency, form a similar microstructure consisting of stoichiometric $\mathrm{TiB}_{2}$ crystalline grains with a pronounced 001 texture encapsulated in amorphous B tissue phase. The crystal plane with the lowest surface energy in $\mathrm{TiB}_{2}$ is the (001)-plane [8]. A 001-texture is thereby expected at growth conditions were adatom mobilities are sufficient to form crystallites with a minimum in surface energy. For the coating deposited using dc sputtering and a floating bias, the $500{ }^{\circ} \mathrm{C}$ substrate temperature does not supply enough energy for a development of 001 texture (see Fig. 2). However, by introducing a $-60 \mathrm{~V}$ bias to the dc sputtering process, sufficient energy is supplied for a 001 texture to develop. Such correlation between texture and bias is often reported in the literature for magnetron sputtered $\mathrm{TiB}_{2}$ coatings $[2,3,10]$. Another approach to supply energy to the growth process while keeping the ion energy at a moderate level is to increase the ion-flux to the substrate. The HiPIMS mode will drastically increase the plasma density and hence the amount of ionized species in the process. As a consequence, a 001 textured coating forms already at $1000 \mathrm{~Hz}$ while retaining a floating bias. The additional energy supplied to the coating surface by reducing the pulse frequency, i.e., increasing the ion-flux to the substrate, or by applying a bias, will not change the texture further since the 001 texture is thermodynamically the most favorable. It is from crosssectional TEM concluded that the coating growth initially are polycrystalline and the 001 orientation outgrows the other orientations after $200-400 \mathrm{~nm}$ (see Fig. 4 and 5). Mikula et al. report a similar trend with a gradual transition from a random to a 001 orientation with the increase in coating thickness [30]. This thickness induced preferred orientation suggest that the orientation and quality of the substrate crystal structure only has a minor or no influence on observed coating texture. Losbichler et al. and Kelesoglu et al. have investigated the separate effect of intensity and energy of ion bombardment by varying the external magnetic field and bias potential during dc magnetron sputtering of $\mathrm{TiB}_{2}$ coatings [9, 12]. Although an increased ion-flux promotes 001 texture, a much stronger dependency with the energy of the bombarding ions (i.e., applied bias), was observed. Thus, a bias was required to achieve a pronounced 001 texture, in contrast to our HiPIMS coatings. It is interesting to note that the FWHM of the 001 diffraction peaks in Fig. 2 remains at about $0.4^{\circ}$ although the pulse frequency is varied. Samuelsson et al. observe an increase in FWHM (i.e., reduced 
crystallinity) with the decrease in pulse frequency for $\mathrm{ZrB}_{2}$ coatings deposited by HiPIMS without any external substrate heating [19]. They argue that a higher degree of ionized species (lower pulse frequency) introduces surface defects, which promotes renucleation and hence smaller grain sizes. In contrast to Samuelsson et al., we use external substrate heating in our study. This will probably suppress the renucleation process and thereby retain the grain size despite the increased degree of ionized species at the lower frequencies. Zhang et al. observe such trend with a smaller influence from the energy of the bombarding ions on crystalline grain size when the substrate temperature is increased for $\mathrm{TiB}_{2}$ coatings deposited by HiPIMS [3].

Since the grains are close to stoichiometric for all coatings, the observed shifts of the 001diffraction peaks are derived from residual stress (see Fig. 2). The expansion of the c-lattice constant with the decrease in pulse frequency correlates to the trend measured for the residual stress with the highest compressive stress determined for the coatings deposited at $200 \mathrm{~Hz}$ and $300 \mathrm{~Hz}$. This correlation is evident from Fig. 3. In the sputtering of $\mathrm{TiB}_{2}$ coatings it is generally observed that compressive stress is induced by applying a bias caused by the incorporation of $\mathrm{Ar}^{+}$ions $[1,2,9,10]$. The high residual stress at the low pulse frequencies is explained by the increased ion-flux to the substrate at these frequencies. The small difference in thermal expansion coefficient perpendicular to the c-axis between the $\mathrm{Al}_{2} \mathrm{O}_{3}$ substrate $(\alpha=$ $\left.7.8 \cdot 10^{-6} \mathrm{~K}^{-1}[21]\right)$ and the $\mathrm{TiB}_{2}$ coating $\left(\alpha=7.0 \cdot 10^{-6} \mathrm{~K}^{-1}[26]\right)$ will only add a small tensile stress component of $210 \mathrm{MPa}$ to the measured residual stress [31]. This in combination with a moderate ion bombardment can probably explain the tensile residual stress measured for the dc sputtered coating at floating bias.

Hardness values above 40 GPa (i.e., superhardness) are measured for all coatings except for the dc sputtered coating using floating bias. As written in the introduction, such high hardness values are typically measured for $\mathrm{TiB}_{2}$ coatings exhibiting a microstructure consisting of 001 textured columnar grains with a B tissue phase in the grain boundaries [7]. Several studies report on the importance of a strong 001 texture for a high hardness [2, 3, 12]. The relatively low hardness (36 GPa) measured for the coating deposited using dc sputtering and a floating bias is therefore explained by the absence of a strong 001 texture for this coating. The hardness increase with the reduction in pulse frequency seen in Fig. 7 is mainly attributed to the higher compressive stress measured for the coatings deposited at the lower frequencies. The stress obstruct dislocation movement resulting in harder coatings. The difference in thickness of the B-tissue phase, as seen by comparing Fig. 4d and Fig. 5d, will probably also influence hardness considering the importance of this phase for superhardness (see discussion in introduction). It should be noted that the effect of residual stress and thickness of tissue phase on hardness cannot be fully separated in this study. The higher density measured for the coatings deposited at the lower frequencies may improve the hardness (see Table I). However, this is most likely a less pronounced effect compared to the residual stress considering the small difference in density between the coatings (see Table I).

The formation of low stress, superhard, and (001) oriented $\mathrm{TiB}_{2}$ coatings has previously been reported for regular dc magnetron sputtered coatings [1, 32]. Two different approaches are used to achieve almost stress free coatings. Berger et al. use a positive bias, which attracts electrons to the substrate during coating growth while Kunc et al. use a combination of $\mathrm{Ar}^{+}$ bombardment and substrate heating. Stress free coatings are formed at substrate temperatures above $400{ }^{\circ} \mathrm{C}$ in both studies. The high degree of ionized species available in HiPIMS enables a possibility to achieve similar superhard 001 textured coatings at low temperatures.

However, this would require extensive experimenting of applied bias and ion-flux and is therefore a separate study in itself. 


\section{Conclusions}

A change from a random oriented polycrystalline structure to a 001 textured nanocolumnar structure is achieved when going from regular dc sputtering to HiPIMS using floating bias. This change in preferred orientation leads to a drastic increase in hardness from $36 \mathrm{GPa}$ to $\geq$ 42 GPa. All coatings deposited by HiPIMS have similar 001 texture, crystallinity, and stoichiometry, independent of pulse frequency and bias mode. An increased ion-flux to the substrate is seen with the decrease in pulse frequency, resulting in a higher residual in-plane biaxial compressive stress as well as denser coatings. The coating deposited by HiPIMS using floating bias and a pulse frequency of $200 \mathrm{~Hz}$ has the highest hardness of $49 \mathrm{GPa}$ as well as the highest compressive stress of -3.8 GPa. We have shown that highly 001 textured and superhard $(\mathrm{H}=43 \pm 1 \mathrm{GPa}) \mathrm{TiB}_{2}$ coatings with a relatively low compressive stress of about $1 \mathrm{GPa}$ can be deposited by HiPIMS at $1000 \mathrm{~Hz}$ using floating bias.

\section{Acknowledgements}

Dr. Grzegorz Greczynski is gratefully acknowledged for assistance with the coating deposition system. The Knut and Alice Wallenberg Foundation is gratefully acknowledged for project funding as well as funding of the Electron Microscopy laboratory in Linköping. J. R. and P. O. Å. P. acknowledges funding from the Swedish Research Council (VR).

\section{References}

[1] M. Berger, L. Karlsson, M. Larsson, S. Hogmark, Low stress $\mathrm{TiB}_{2}$ coatings with improved tribological properties, Thin Solid Films 401 (2001) 179-186.

[2] M. Mikula, B. Grancic, V. Bursikova, A. Csuba, M. Drzik, S. Kavecky, A. Plecenik, P. Kus, Mechanical properties of superhard $\mathrm{TiB}_{2}$ coatings prepared by $\mathrm{DC}$ magnetron sputtering, Vacuum 82 (2007) 278-281.

[3] T.F. Zhang, B. Gan, S.-m. Park, Q.M. Wang, K.H. Kim, Influence of negative bias voltage and deposition temperature on microstructure and properties of superhard $\mathrm{TiB}_{2}$ coatings deposited by high power impulse magnetron sputtering, Surf. Coat. Technol. 253 (2014) 115122.

[4] N. Schalk, J. Keckes, C. Czettl, M. Burghammer, M. Penoy, C. Michotte, C. Mitterer, Investigation of the origin of compressive residual stress in $\mathrm{CVD} \mathrm{TiB}_{2}$ hard coatings using synchrotron X-ray nanodiffraction, Surf. Coat. Technol. 258 (2014) 121-126.

[5] C. Pfohl, A. Bulak, K.T. Rie, Development of titanium diboride coatings deposited by PACVD, Surf. Coat. Technol. 131 (2000) 141-146.

[6] P. Vajeeston, P. Ravindran, C. Ravi, R. Asokamani, Electronic structure, bonding, and ground-state properties of $\mathrm{AlB}_{2}$-type transition-metal diborides, Phys. Rev. B 63 (2001) 045115.

[7] P.H. Mayrhofer, C. Mitterer, J.G. Wen, J.E. Greene, I. Petrov, Self-organized nanocolumnar structure in superhard $\mathrm{TiB}_{2}$ thin films, Appl. Phys. Lett. 86 (2005) 131909.

[8] J. Pelleg, G. Sade, M. Sinder, D. Mogilyanski, Compositional and structural changes in $\mathrm{TiB}_{2}$ films induced by bias, in situ and post-deposition annealing, respectively, Phys. B Cond. Matt. 381 (2006) 118-127. 
[9] P. Losbichler, C. Mitterer, Non-reactively sputtered TiN and $\mathrm{TiB}_{2}$ films: influence of activation energy on film growth, Surf. Coat. Technol. 97 (1997) 567-573.

[10] R. Lohmann, E. Osterschulze, K. Thoma, H. Gartner, W. Herr, B. Matthes, E. Broszeit, K.H. Kloos, Analysis of r.f.-sputtered $\mathrm{TiB}_{2}$ hard coatings by means of X-ray diffractometry and Auger electron spectroscopy, Mater. Sci. Eng. A 139 (1991) 259-263.

[11] J. Neidhardt, S. Mraz, J.M. Schneider, E. Strub, W. Bohne, B. Liedke, W. Moller, C. Mitter, Experiment and simulation of the compositional evolution of Ti-B thin films deposited by sputtering of a compound target, J. Appl. Phys. 104 (2008) 063304.

[12] E. Kelesoglu, C. Mitterer, M.K. Kazmanli, M. Urgen, Microstructure and properties of nitride and diboride hard coatings deposited under intense mild-energy ion bombardment, Surf. Coat. Technol. 116 (1999) 133-140.

[13] N. Kalfagiannis, G. Volonakis, L. Tsetseris, S. Logothetidis, Excess of boron in $\mathrm{TiB}_{2}$ superhard thin films: a combined experimental and ab initio study, J. Phys. D Appl. Phys. 44 (2011) 385402.

[14] K. Sarakinos, J. Alami, S. Konstantinidis, High power pulsed magnetron sputtering: A review on scientific and engineering state of the art, Surf. Coat. Technol. 204 (2010) 16611684.

[15] J.T. Gudmundsson, N. Brenning, D. Lundin, U. Helmersson, High power impulse magnetron sputtering discharge, J. Vac. Sci. Technol. A 30 (2012) 030801.

[16] I. Petrov, P.B. Barna, L. Hultman, J.E. Greene, Microstructural evolution during film growth, J. Vac. Sci. Technol. A, 21 (2003) S117-S128.

[17] A.P. Ehiasarian, A. Vetushka, Y.A. Gonzalvo, G. Safran, L. Szekely, P.B. Barna, Influence of high power impulse magnetron sputtering plasma ionization on the microstructure of TiN thin films, J. Appl. Phys. 109 (2011) 104314.

[18] J. Bohlmark, J. Alami, C. Christou, A.P. Ehiasarian, U. Helmersson, Ionization of sputtered metals in high power pulsed magnetron sputtering, J. Vac. Sci. Technol. A 23 (2005) 18-22.

[19] M. Samuelsson, J. Jensen, U. Helmersson, L. Hultman, H. Högberg, $\mathrm{ZrB}_{2}$ thin films grown by high power impulse magnetron sputtering from a compound target, Thin Solid Films 526 (2012) 163-167.

[20] G. Janssen, M.M. Abdalla, F. van Keulen, B.R. Pujada, B. van Venrooy, Celebrating the 100th anniversary of the Stoney equation for film stress: Developments from polycrystalline steel strips to single crystal silicon wafers, Thin Solid Films 517 (2009) 1858-1867.

[21] T. Vodenitcharova, L.C. Zhang, I. Zarudi, Y. Yin, H. Domyo, T. Ho, M. Sato, The effect of anisotropy on the deformation and fracture of sapphire wafers subjected to thermal shocks, J. Mater. Process. Technol. 194 (2007) 52-62. 
[22] J.M. Winey, Y.M. Gupta, D.E. Hare, r-axis sound speed and elastic properties of sapphire single crystals, J. Appl. Phys. 90 (2001) 3109-3111.

[23] W.C. Oliver, G.M. Pharr, An improved technique for determining hardness and elasticmodulus using load and displacement sensing indentation experiments, J. Mater. Res. 7 (1992) 1564-1583.

[24] A.C. Fischer-Cripps, Review of analysis and interpretation of nanoindentation test data, Surf. Coat. Technol. 200 (2006) 4153-4165.

[25] I.R. Shein, A.L. Ivanovskii, Elastic properties of mono- and polycrystalline hexagonal $\mathrm{AlB}_{2}$-like diborides of s, $\mathrm{p}$ and $\mathrm{d}$ metals from first-principles calculations, J. Phys. Condes. Matter 20 (2008) 415218.

[26] R.G. Munro, Material properties of titanium diboride, J. Res. Natl. Inst. Stand. Technol. 105 (2000) 709-720.

[27] Y. Mu, K. Chen, W.J. Meng, F. Mei, Low temperature deposited titanium boride thin films and their application to surface engineering of microscale mold inserts, Microsyst Technol 18 (2012) 667-677.

[28] C. Ruby, R. Ott, F. Huang, M.L. Weaver, J.A. Barnard, XPS study of reactively sputtered Ti-B-N hard coatings, Surf. Interface Anal. 29 (2000) 823-828.

[29] X. Ma, C. Li, Z. Du, W. Zhang, Thermodynamic assessment of the Ti-B system, J Alloys Compd. 370 (2004) 149-158.

[30] M. Mikula, B. Grančič, T. Roch, T. Plecenik, I. Vávra, E. Dobročka, A. Šatka, V. Buršíková, M. Držík, M. Zahoran, A. Plecenik, P. Kúš, The influence of low-energy ion bombardment on the microstructure development and mechanical properties of $\mathrm{TiB}_{\mathrm{x}}$ coatings, Vacuum 85 (2011) 866-870.

[31] M. Ohring, Materials Science of Thin Films, 2nd ed., Academic Press, San Diego, 2001, 732-733.

[32] F. Kunc, J. Musil, P.H. Mayrhofer, C. Mitterer, Low-stress superhard Ti-B films prepared by magnetron sputtering, Surf. Coat. Technol. 174-175 (2003) 744-753. 
